\title{
On the Diagnosticity of Motyl et al. (in press).
}

By: Matt Motyl, Linda J. Skitka, \& Alex Demos

May 7, 2017

We appreciate Uri Simonsohn, Joe Simmons, and Leif Nelson's recent critique and the opportunity to review it before they posted it. We welcome all critiques and commentaries as we believe that this is how the scientific method should work, and the critiques help us to advance knowledge. We also appreciate that Leif Nelson disclosed that he pre-registered a very similar project to ours (see Footnote 1 in Data Colada blog; his pre-registration from December 8, 2015 is here, our pre-registration from May 26, 2015 is here), that involves manually coding papers published in JPSP to address similar questions about evidentiary value from 2006-2015. The fact that multiple labs, unbeknownst to each other, are undertaking such similar projects highlights the importance and timeliness of the questions: What is the status of social/personality science? And, how do we best establish the evidentiary value of research in the field?

Simonsohn, Simmons, and Nelson, however raised some concerns about the quality of our manual coding of test statistics in the more than 1,800 studies we coded for our project (in press at JPSP). If the coding issues proposed by Simonsohn, Simmons, and Nelson are correct and characterize our full data set, the worst case scenario is that our report is based on randomly selected reported statistics (e.g., some manipulation checks, omnibus tests, follow-up tests, etc.). Then what we have is akin to machine-scraped $p$-values--no more, no less. To the extent that scraped $p$-values have been widely used to make inferences about the status of our science (e.g., De Winter \& Dodou, 2015; Head, Holman, Lanfear, Kahn, \& Jennions, 2015; Nuijten, Hartgerink, van Assen, Epskamp, \& Wicherts, 2016), our data are (at a minimum) at least as valuable as scraped data for making claims about the status of our science.

That said, there are reasons to believe we were not randomly selecting focal statistical tests, and to be skeptical of a conclusion that our coding methods were flawed based on a very small $(N=$ 10 , or $0.5 \%$ ) and non-random sample of the more than 1800 coded focal tests from our full data set, using unclear sampling procedures. Neither paper in our sample by Sanna (one of which was re-coded by Simonsohn, Simmons, and Nelson) was rated as "very easy" to code. Another paper we recognized in the blog (Gollwitzer et al., given one of us was an author on it) was not in the top 10 of the file when sorted by coding ease (in fact, it was \#275). To some extent, we can't help but feel like this blog is making use of the availability heuristic (Tversky \& Kahneman, 1973) to make their argument, rather than making an argument based on a proper systematic resampling of our work.

In addition, Simonsohn, Simmons, and Nelson did not consistently conform to our coding guidelines when they claim we "mis-coded" certain values. As can be seen in our instructions to coders (link), we made the decision to code omnibus values when the key hypothesis test was an 
interaction. Coding omnibus terms was not a coding error: It was by design and correct code in accordance with our coding instructions ${ }^{\mathrm{i}}$. These codes are therefore not "errors," but instead reflect a disagreement in judgment about how to best represent tests of hypothesized interactions. Others are welcome to code using other decision rules and see if the results differ, but this criticism alone does not undermine accuracy of our coding.

Another major concern raised by this blog post was the integrity and potential experience of our coders. We note that our coders each had training in advanced statistics, and were extensively trained. The majority of our coders ${ }^{\mathrm{ii}}$ and the lead author initially worked together for two weeks, approximately 8 hours per day on the same set of studies, and coded to consensus (an effort that required massive infusions of pizza, gourmet Mac and Cheese, and coffee to endure), and through this process developed a set of coding rules and guidelines. After the two-week period, coders were randomly assigned a set of independent papers to code. At the end of coding independently, we tested for inter-coder agreement with a set of randomly assigned studies. There was $80.2 \%$ agreement on the focal test statistic, a generally acceptable level of agreement/reliability. To further test whether our coding has integrity, we tested whether we observed different results as a function of coder. A quick examination of a few of the metrics, revealed that the confidence intervals overlapped across coders for R-Index, sample size, and median $Z$-scores. We should note: This coding was not easy and we are not claiming perfection; there are certainly some coding errors in our data. That said, we do claim that our data meets customary standards for quality in open-ended coding and that steps were taken to ensure appropriate standards of data quality for a project of this type (e.g., careful training and quality checks).

Simonsohn, Simmons, and Nelson also imply that our coding must have been impossibly too quick and dirty to be valid. Based on their own manual coding efforts, they argue that it should have taken approximately 3 hours to thoughtfully code a single paper. This conclusion is consistent with our own experience: Our coders took between 30 and 60 minutes per study (depending on its complexity) to code the key test statistic. Papers in 2003-2004 reported on an average of 2.39 studies and 3.15 studies in 2013-2014 ${ }^{\text {iii }}$. As a team we calculate that we spent roughly 1,800 hours coding studies. There are reasons this paper has many authors: It really took a village. Although there will always be some coding errors in any project of this scope and complexity, it would be wrong to claim that our effort was anything close to hasty.

Moreover, Uli Schimmack was also interested in our paper and results. He independently verified our conclusions with respect to the R-Index, Z-curve, and TIVA, and compared them to the Open Science Collaboration manually-coded statistics that were heavily reviewed and vetted just for social psychology studies and finds they are similar (see his blog and the graphs below for his major findings). Specifically, Schimmack concludes that his "....independent replication study closely replicates the estimates obtained with Motyl et al.'s data." Schimmack, however, 
took issue with our conclusion that the R-Index specifically suggested the field was rotten: He interpreted our R-Index findings to be more consistent with the conclusion against rottenness, and as evidence of (at least some) evidentiary value in social and personality psychology, an interpretation consistent with most of the other indices we calculated. All these factors together lead us to continue to have reasonable confidence in our data.
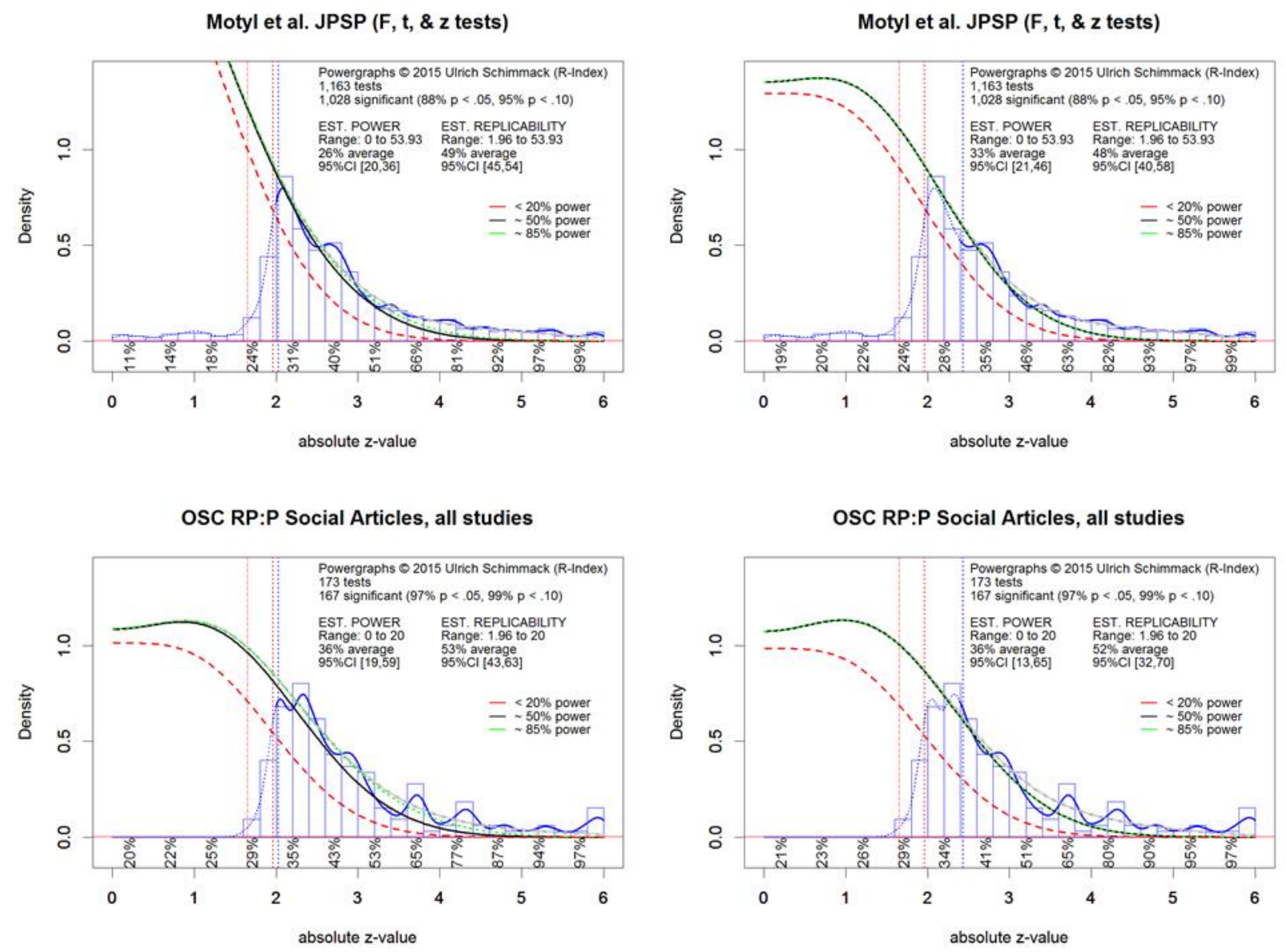

Finally, it might be useful for others to know that our project is the fruit of a very friendly, but nonetheless genuinely adversarial collaboration. The priors of our group about what we expected to find ranged from truly deep pessimism to very cautious optimism about the status of our science (both historically and in the present), as well as many positions between these two end points. As a group, we genuinely wanted to find out what the data had to say and to gain better insight on where the field was prior to the status of our science discussion, as well as where it is headed as a consequence. Our interpretation of our results comes from negotiating from these very different starting points, something we think is an important strength of our approach and orientation. Moreover, as a group, we have no personal attachment or investment in any given index of evidentiary value: We made use of every index we reasonably could, again, in an attempt to open-mindedly explore the evidentiary status of our field. These indices are still in 
their infancy, and we think our project reveals that we still have a lot of work to do to better understand how to estimate (and even precisely define what counts as) evidence of evidentiary value and replicability. Our effort is meant to be a preliminary step forward, but hardly the definitive word on these issues.

In conclusion, our paper is not meant to be the final answer on the status of our science, but rather one step towards better understanding the status of our science. We welcome Uri, Joe, and Leif's independent replication and extension of our findings, given they have embarked on a similar coding effort. Additionally, all of our data, materials, and scripts have been publicly available since before we submitted our paper for review (where we received feedback from 1 editor and 5 peer reviewers, with 2 rounds of revision; we also received feedback from several other scholars prior to submission). We invite all interested parties to review our materials and do any additional analyses of interest. It is our hope that in 10 years, this paper will look archaic as we come to a better understanding of how to estimate evidentiary value; we hope that others will take this data-driven approach, expand it, and improve upon it.

\section{References}

de Winter, J. C., \& Dodou, D. (2015). A surge of p-values between 0.041 and 0.049 in recent decades (but negative results are increasing rapidly too). PeerJ, 3, e733. http://dx.doi.org/10.7717/peerj.733

Head, M. L., Holman, L., Lanfear, R., Kahn, A. T., \& Jennions, M. D. (2015). The extent and consequences of p-hacking in science. PLoS Biol, 13(3), e1002106.

Nuijten, M. B., Hartgerink, C. H., van Assen, M. A., Epskamp, S., \& Wicherts, J. M. (2016). The prevalence of statistical reporting errors in psychology (1985-2013). Behavior research methods, 48(4), 1205-1226.

Tversky, A., \& Kahneman, D. (1973). Availability: A heuristic for judging frequency and probability. Cognitive psychology, 5(2), 207-232.

\footnotetext{
i Simonsohn, Simmons, and Nelson were somewhat inconsistent in what they seemed to argue should be coded in the case of predicted interactions. On the one hand, they implied that we should have coded the one simple comparison, and that sometimes we coded the wrong one. On the other hand, they also lamented that some authors do not report the omnibus interaction term (a serious omission), which implies the omnibus test provides important information and may therefore be the more important focal test statistic when authors predict an interaction. Any given simple comparison can be significant even in the absence of an interaction; a simple comparison by itself therefore seems incomplete evidence of a proposed interaction. We therefore thought it was more important to code whether the hypothesized interaction was significant when authors predicted an interaction (and not specific simple comparisons), because this test statistic would better capture whether the effect of a focal IV was in fact moderated by the proposed moderator. That our coders coded omnibus interaction terms was therefore a designed feature, and not a bug, of our coding scheme.

ii Two coders participated remotely in this training, and one coder joined the project after this initial period, but was extensively briefed on the coding scheme.

iii Simonsohn, Simmons, and Nelson are also focusing their current project on coding articles in JPSP, which tend to have more studies than articles in Psychological Science, JESP, and PSPB. Therefore, their time estimate likely is very similar to ours, when considering the number of studies per paper being coded.
} 